\title{
Changing environment against duplicate publication
}

\author{
Shigeru Ehara
}

Received: September 6, 2008 / Accepted: September 16, 2008

(C) Japan Radiological Society 2009

Duplicate publication is defined as unauthorized reproduction of an author's own publication, and it is scientific misconduct that is considered unethical. In the past, publication of the same material in different languages was considered more acceptable because the distribution of the publications was limited to a small medical community. The thesis for the Doctor of Medical Science degree, a unique postgraduate degree in Japan, published in the journals of medical schools or local medical societies in Japanese, may be republished in English by the same or a different member of the research group. The readers of papers were different, and such redundant publication helped disseminate knowledge in groups with different native languages and specialties. However, the environment around publication has changed significantly. We now have an enormous flow of information, and redundant publications have overwhelmed our capacity for control. Those of us who access databases regularly face a huge volume of redundant information.

We may still have a need to publish the same or similar materials, but due course should be followed. ${ }^{1}$ The editors of primary and secondary publications should agree on the process, and the priority and content of the primary publication should be respected by mentioning that publication and including it in the references. There have been some differences in the authorization policy toward reproducing the same material among journals. Some surgical journals still allow reproduction of the

\footnotetext{
S. Ehara $(\bowtie)$

Department of Radiology, Iwate Medical University, 19-1 Uchimaru, Morioka 020-8505, Japan

Tel. +81-19-651-5111 (ext. 3689); Fax +81-19-651-7071

e-mail: erhara@iwate-med.ac.jp
}

same materials in different languages. In the field of radiology, however, the policy against duplicate publication has been generally tough. The American Journal of Roentgenology raised the issue of redundant publication in different languages in 1999 and stated that the use of the same materials in different languages would be considered duplicate publication. ${ }^{2}$

Duplicate publications, which are numerous, are efficiently detected. Many publications in Japanese are now indexed and incorporated in an English-language database even if no English document is included in the original paper. Titles and abstracts of many Japanese publications can now be sought in the database, and they can be subjects of investigation for redundant publications. Even with a few key words, we can detect similar papers quite easily. Among them, papers by the same authors, dealing with similar or the same hypotheses and subjects, can be easily suspected to be duplicate publications. A group in France used MEDLINE to investigate redundant publications and identified such publications based on the similarities in the hypothesis, number of subjects, results, at least one common author, and no new information. ${ }^{3}$ The Déjà $\mathrm{Vu}$ database of the University of Texas Southwestern Medical School, funded by the Hudson Foundation and the National Institutes of Health, has accumulated and incorporated a large number of duplicate publications that can be accessed. ${ }^{4}$

Publications by Japanese investigators tend to be thought to contain more redundant information. In the current issue of Nature, based on the MEDLINE and Déjà $\mathrm{Vu}$ databases, the contribution to science and possible redundant publication was viewed country by country. ${ }^{5}$ Although the overall contribution of the Japanese scientific community has proved to be high, 
Japanese literature was also reported to have a high rate of duplicate (or similar) publications - a rate that is almost twice as high as in other countries. ${ }^{5}$

This analysis is still performed manually, but automatic analysis may well be seen in the near future. Digital technology has not only accelerated the speed of publication, but digital data analysis provides us further insight into the content of publications. Automatic search engines have enabled the analysis of a large volume of radiology documents. A group in Boston analyzed more than 4 million radiology reports regarding the tendency to recommend further examinations. ${ }^{6}$ As automatic search engines have been enabled to assess the pattern of radiology practice, similar or the same approaches will be applicable to detect duplicate publications. Automatic search engines may well detect similarities in publications faster and more easily. In addition, although automatic translation software between Japanese and most Western languages is still in its infancy, more sophisticated translation software will soon expand resource databases in different languages.
In this situation, we have to tighten the policy against duplicate publication to avoid the bad reputation of a high duplicate publication rate by Japanese investigators. It is time to revise our curriculum vita to avoid being suspected of redundant publications, which may be more easily detected in the future.

\section{References}

1. Berquist TH. Duplicate publishing or journal publication ethics 101. AJR Am J Roentgenol 2008;191:311-2.

2. Rogers LF. In any language. AJR Am J Roentgenol 2000; 174:1487.

3. Arrive L, Lewin M, Dono P, Monnier-Cholley L, Hoeffel C, Tubiana J-M. Redundant publication in the journal Radiology. Radiology 2008;247:836-40.

4. https://spore.swmed.edu/dejavu

5. Errami M, Garner H. A tale of two citations. Nature 2008; 451:397-9.

6. Dang PA, Kalra MK, Blake MA, Schultz TJ, Halpern EF, Dreyer K. Extraction of recommendation features in radiology with natural language processing: exploratory study. AJR Am J Roentgenol 2008;191:313-20. 